\title{
Prevention of Potential Adverse Metabolic Effects of a Supplementation with Omega-3 Fatty Acids Using a Genetic Score Approach
}

\author{
Maximilien Franck ${ }^{a, b}$ Juan de Toro-Martín ${ }^{a, b}$ Frédéric Guénard ${ }^{a, b}$ Iwona Rudkowska ${ }^{c, d}$

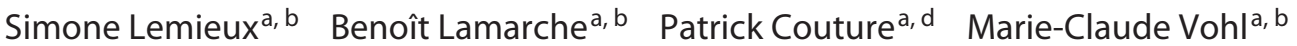 \\ anstitute of Nutrition and Functional Foods (INAF), Laval University, Quebec City, QC, Canada; b School of Nutrition, \\ Laval University, Quebec City, QC, Canada; 'Department of Kinesiology, Laval University, Quebec City, QC, Canada; \\ dEndocrinology and Nephrology Unit, CHU de Quebec Research Center, Quebec City, QC, Canada
}

\section{Keywords \\ Genome-wide association study - Genetic risk score • \\ Precision nutrition - Omega-3 fatty acids · Insulin resistance · Glycemic control · Metabolic homeostasis}

\begin{abstract}
Introduction: The consumption of long-chain omega-3 polyunsaturated fatty acids ( $\mathrm{n}-3$ PUFA) has been reported to have beneficial health effects, notably, by reducing plasma triglyceride levels. Nonetheless, a concomitant decrease in insulin sensitivity has also been observed, but is highly variable among subjects. Herein, we aimed to determine the importance of the genetic background in the interindividual variability of the insulin sensitivity response following an $n-3$ PUFA supplementation. Methods: A total of 210 participants completed a 6-week n-3 PUFA supplementation with $5 \mathrm{~g} /$ day of fish oil (providing 1.9-2.2 g of eicosapentaenoic acid $+1.1 \mathrm{~g}$ of docosahexaenoic acid). Insulin resistance was estimated by the homeostatic model assessment (HOMA-IR), and participants were further classified as high-risk or lowrisk depending on their HOMA-IR change following the $n-3$ PUFA supplementation, as compared to pre-supplementation values. Genome-wide genotyping data were obtained
\end{abstract}

for 138 participants using HumanOmni-5-Quad BeadChips containing 4,301,331 single nucleotide polymorphisms. A genome-wide association analysis (GWAS) was carried out between high-risk and low-risk participants. The population study was split into training (60\%) and testing (40\%) datasets to assess the predictive accuracy of a genetic risk score (GRS) constructed by summing the number of risk alleles. Results: Following the n-3 PUFA supplementation, 32 participants had increased HOMA-IR as compared to initial values and were classified as high risk (23.2\%), whereas remaining subjects were classified as low risk ( $n=106,76.8 \%)$. A total of 8 loci had frequency differences between high-risk and lowrisk participants at a suggestive GWAS association threshold ( $p$ value $<1 \times 10^{-5}$ ). After applying 10 -fold cross validation, the GRS showed a significant association with the risk of increased HOMA-IR in the testing dataset $(\mathrm{OR}=3.16[95 \% \mathrm{Cl}$, 1.85-7.14]), with a predictive accuracy of 0.85 , and explained $40 \%$ of variation in HOMA-IR change. Conclusions: These results suggest that the genetic background has a relevant role in the interindividual variability observed in the insulin sen-

Maximilien Franck and Juan de Toro-Martín contributed equally to this work.

\begin{tabular}{|c|c|}
\hline KARGER & $\begin{array}{l}\text { (c) } 2019 \text { The Author(s) } \\
\text { Published by S. Karger AG, Basel }\end{array}$ \\
\hline $\begin{array}{l}\text { E-Mail karger@karger.com } \\
\text { www.karger.com/lfg }\end{array}$ & $\begin{array}{l}\text { This article is licensed under the Creative Commons Attribution- } \\
\text { NonCommercial-NoDerivatives } 4.0 \text { International License (CC BY- } \\
\text { NC-ND) (http://www.karger.com/Services/OpenAccessLicense). } \\
\text { Usage and distribution for commercial purposes as well as any dis- } \\
\text { tribution of modified material requires written permission. }\end{array}$ \\
\hline
\end{tabular}

Marie-Claude Vohl

Institute of Nutrition and Functional Foods (INAF), Laval University

2440 Boulevard Hochelaga Suite 1710

Quebec City, QC G1V 0A6 (Canada)

E-Mail marie-claude.vohl@ fsaa.ulaval.ca 
sitivity response following an n-3 PUFA supplementation. Subjects being at risk of insulin sensitivity lowering following an n-3 PUFA supplementation may be identified using genetic-based precision nutrition approaches.

(C) 2019 The Author(s)

Published by S. Karger AG, Basel

\section{Introduction}

The human metabolism has been subjected to evolutionary pressures by the dietary environment, which have progressively shaped its genetic architecture, leading to variable frequencies of some genetic variants in metabolic genes [1-7]. The genetic background may to some extent explain both the maladaptation to the modern Western diet, leading to metabolic diseases and to the heterogeneous response to different nutrients, including long-chain omega-3 polyunsaturated fatty acids (n-3 PUFA) $[4,8]$.

Glycemic control is a key feature of metabolic homeostasis, which depends on insulin sensitivity to maintain a healthy state [9]. Given that, both dyslipidemia and altered glycemic control are major risk factor for cardiovascular diseases, there is a need to identify therapeutic strategies to restore metabolic homeostasis. In that respect, nutritional interventions premised upon specific nutrients or bioactive compounds are emerging as tangible strategies, although questions remain surrounding their interaction with the genetic background [10]. This is the case for n-3 PUFA, whose effectiveness in treating and preventing several features of the metabolic syndrome, mostly relying on their anti-inflammatory and hypolipidemic actions, has been extensively reported [11-14]. Importantly, previous studies have also shown that part of the large heterogeneity observed in the response to an $n-3$ PUFA supplementation depends on the genetic background [15-17]. Although beneficial effects of n-3 PUFA on cardiovascular health depend on several reported actions not necessarily involving a triglyceride (TG)-lowering effect [18], part of the heterogeneity observed in the response to n-3 PUFA has been attributed to a highly variable plasma TG response, that can now be predicted using genetic risk scores (GRS) [3, 16, 19]. Similarly, heterogeneous effects of n-3 PUFA on insulin sensitivity and glucose homeostasis across different cohorts have also been reported [3, 20, 21].

Genetic determinants of such interindividual variability in the metabolic response to n-3 PUFA supplementation should be accounted for within a perspective of per-

Insulin Sensitivity Lowering following an n-3 PUFA Supplementation sonalized or gene-based nutrition $[10,14]$. In this regard, unlike vigorous research concerning the genetic features involved in the variability of lipid homeostasis, studies that evaluate the relationship between n-3 PUFA supplementation and the associated glycemic response with regard to the interindividual genetic variability are still very scarce [21]. The aim of the present study was to determine the relevance of the genetic background in the interindividual variability of the insulin sensitivity response following an n-3 PUFA supplementation.

\section{Materials and Methods}

\section{Subjects and Study Design}

This work is based on the Fatty Acid Sensor (FAS) Study, a sample of volunteers recruited in the Quebec City metropolitan area (registered at ClinicalTrials.gov as NCT01343342). Briefly, 254 subjects with a body mass index (BMI) ranging from 25 to 40 $\mathrm{kg} / \mathrm{m}^{2}$ were recruited in order to examine the plasma TG response to a 6-week n-3 PUFA supplementation. A detailed description of the methodology of the FAS Study, including screening, recruitment procedures, laboratory methods, and statistical analysis, has been reported elsewhere [16]. From the 254 participants, 210 completed the 6-week n-3 PUFA supplementation with 5 g/day of fish oil (providing 1.9-2.2 g of eicosapentaenoic acid [EPA] $+1.1 \mathrm{~g}$ of docosahexaenoic acid [DHA]). Besides, in order to illustrate the heterogeneous insulin sensitivity response to an n-3 PUFA supplementation, participants from the ComparED Study [22] were herein used for comparison purposes. The ComparED Study is a double-blind, randomized, controlled, crossover design with three 10 -week treatment arms, where 154 participants received $2.7 \mathrm{~g}$ of: (1) EPA, (2) DHA, and (3) corn oil (placebo). The FAS Study and the ComparED Study both recruited healthy subjects from the greater Quebec City metropolitan area using the clinical setting of the Institute of Nutrition and Functional Foods (INAF) of Laval University; consequently, we can assume that there are no major differences in ethnicity, genetics, diet, and lifestyle habits. Moreover, biochemical data have been measured for both studies in the clinical biochemistry laboratory of the Laval University Hospital Research Center (CHUL-CHUQ) using the exact same methods. The fatty acid content change in erythrocyte phospholipids after the n-3 PUFA supplementation in the FAS Study and the ComparED Study has been previously reported $[22,23]$.

\section{Patient Stratification}

Previously, the 210 participants who completed the FAS Study were stratified into TG responders and nonresponders [16]. Subjects showing no reduction, or an increase, in plasma TG levels between pre- and post-supplementation were defined as nonresponders, whereas those showing a decrease in plasma TG levels ( $\Delta \mathrm{TG} \geq 0.01 \mathrm{mM}$ ) were defined as responders. From the total of 210 participants, 141 showed a TG response higher than a threshold based on the technical error of measurement (TEM) [24] and were classified as extreme TG responders [25]. From these, 138 participants with available pre- and post-supplementation plasma glucose and insulin levels were stratified according 
to insulin resistance status and included for further analyses in the present study. Insulin resistance was estimated before and after the n-3 PUFA supplementation by the homeostatic model assessment (HOMA-IR). High-risk and low-risk participants were defined according to the HOMA-IR change between preand post-supplementation states. For each subject, two HOMAIR measurements from screening and pre-supplementation visits were used to determine the TEM: a threshold value ensuring an accurate distinction between HOMA-IR variations due to $n-3$ PUFA supplementation and those due to intraindividual variability [24]. Participants were further classified as high-risk when they exhibited an HOMA-IR variation above the TEM threshold and low-risk when HOMA-IR variation was within or below the TEM range in response to the n-3 PUFA supplementation. The calculation of TEM in the ComparED Study was performed by using pre- and post-supplementation HOMA-IR values in the placebo arm.

\section{Single Nucleotide Polymorphism Genotyping and Quality}

Control

Illumina HumanOmni-5-Quad BeadChip ${ }^{\circledR}$ (Illumina Inc., San Diego, CA, USA) were used to genotype 4,301,331 single nucleotide polymorphisms (SNPs) at the genome-wide level in the 138 individuals. Genotyping arrays were processed at the McGill University/Génome Québec Innovation Center (Montreal, QC, Canada) according to manufacturer's recommendations. Quality control procedures were applied: minor allele frequency $>1 \%$, deviations from Hardy-Weinberg equilibrium $(p$ value $\left.<1.87 \times 10^{-8}\right)$, call rate $(>95 \%)$, and gender mismatch were verified based on genotyping data. Monoallelic SNPs were also removed leading to the exclusion of $1,632,526$ SNPs. In this manner, a total of $2,668,805$ SNPs were available for statistical analyses.

\section{Genetic Risk Score}

Allele frequencies were compared between high-risk and lowrisk participants using PLINK (https://www.cog-genomics.org/ plink2) [26]. The ratio between high-risk participants having a specific allelic variant and low-risk participants having the same allele was determined. In addition, a $p$ value was calculated using a $\chi^{2}$ test in order to define the statistical significance of the odds ratio (OR) with $95 \%$ confidence intervals $(95 \% \mathrm{CI})$. The threshold for genome-wide significance was set at Bonferroni-adjusted $p$ value $<$ $1.87 \times 10^{-8}(0.05 / 2,668,805)$, and suggestive significance was defined as $p$ value $<1 \times 10^{-5}$, as previously proposed for the analysis of complex traits, where a number of SNPs are expected to contribute to the variance of the trait [27]. For each significant locus, lead SNPs were determined by the linkage disequilibrium (LD) clumping approach in PLINK (SNPs located less than $250 \mathrm{~kb}$ away from an index variant and LD $r^{2}>0.8$ ). The GRS was constructed according to the additive effects model. All the SNPs associated with an increase in HOMA-IR following the n-3 PUFA supplementation, i.e. with the minor allele being more represented in the high-risk group, were encoded as 0 when homozygous for major allele, 1 when heterozygous, and 2 when homozygous for minor allele. The SNPs associated with a decrease in HOMA-IR were assigned using the same coding but with a negative sign. A GRS was finally calculated for each study participant by summing the number of alleles of each risk SNP.
Statistical Analyses and Evaluation of GRS Performance

The predictive power of the GRS was assessed by binomial logistic regression. The $\mathrm{R}$ caret package was used to train, cross-validate, and test the model $[28,29]$. The population study was split randomly in training (60\%) and testing (40\%) datasets, with a balanced ratio of high-risk and low-risk participants in each dataset. A 10-fold cross-validation was performed in the training dataset. Cross-validation consisted in partitioning the training dataset into 10 folds that were one at a time alternately omitted from the dataset before prediction test with the remaining units. The prediction procedure was computed for each training dataset composed of 9 of the units and repeated until every unit has been removed once. Errors observed within each partition were averaged to provide the overall cross-validation error. The final model with adjusted parameters obtained in the training dataset was further assessed in the testing dataset. The area under the receiver operating characteristic curve (AUC-ROC) was used to evaluate the predictive performance of the GRS. The accuracy, corresponding to the proportion of true results in a population (number of correct high-risk and low-risk assessments/total number of assessments), was also used to evaluate the degree of veracity of the GRS. The McFadden's pseudo $R^{2}$ was used to estimate the proportion of explained variation in the response (HOMA-IR variation) accounted for by the model (GRS). The McFadden's pseudo $R^{2}$ is computed as the ratio between the log-likelihood value for the model containing the predictors and the log-likelihood value for the intercept-only model [30]. All statistical analyses were performed in R [31].

\section{Results}

\section{n-3 PUFA Supplementation Led to Lowering of}

Insulin Sensitivity in 23\% of Subjects

The heterogeneity in individual HOMA-IR variation following the n-3 PUFA supplementation is presented in Figure 1. Following the n-3 PUFA supplementation, the HOMA-IR decreased or remained within the TEM range in 106 participants (low risk $=76.8 \%$ ), whereas 32 subjects (high risk $=23.2 \%$ ) demonstrated an increased HOMA-IR beyond the TEM threshold value (TEM = 0.66) (Fig. 1a). More precisely, high-risk subjects showed an average post-supplementation increase in HOMA-IR values of $57.2 \%$. For comparison purposes, individual changes in HOMA-IR following an EPA or DHA supplementation from the ComparED Study [22] have been put into perspective. First, we found that the calculated TEM was on the same scale as in the FAS Study (TEM $=0.68$ ). Second, the overall decrease in insulin sensitivity observed in the FAS Study (23.2\%) was comparable to that observed in both DHA (18.9\%) (Fig. 1b) and EPA (25.8\%) (Fig. 1c) arms in the ComparED Study. The light thrown on this interindividual variability following n-3 PUFA supplementation led to the stratification of subjects according to their insulin sensitivity change. 

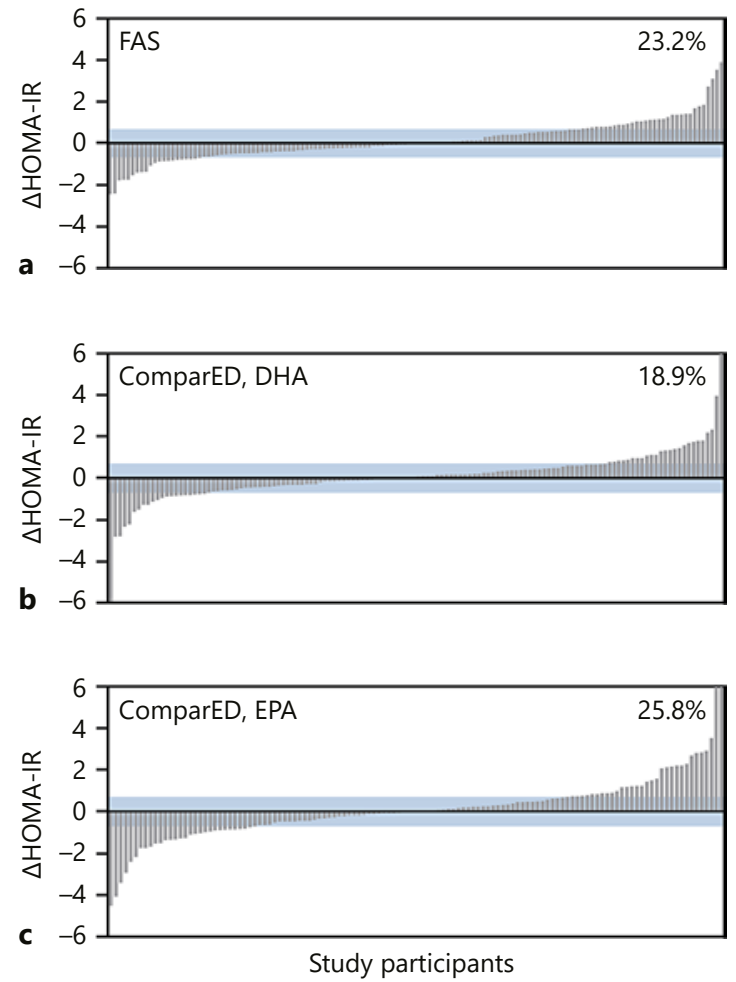

Fig. 1. Individual changes in HOMA-IR following n-3 PUFA supplementation. The $y$ axis represents the HOMA-IR change between pre- and post-supplementation values in participants from the FAS Study (a), and in participants from the docosahexaenoic acid (DHA) and eicosapentaenoic acid (EPA) arms of the ComparED Study (b and $\mathbf{c}$, respectively). Each bar on the $x$ axis represents 1 participant. High-risk and low-risk participants were determined based on the technical error of measurement (TEM; blue bracket). Participants with HOMA-IR change above the TEM threshold were classified as high risk, whereas participants with HOMA-IR change within or below the TEM range were classified as low risk. The percentage of high-risk participants is shown.

\section{High-Risk Participants Showed Higher HOMA-IR following n-3 PUFA Supplementation}

No significant differences were found in the proportion of men and women between high-risk and low-risk groups (Table 1). Participants from the high-risk group were significantly younger than low-risk participants $(28.8 \pm 8.4$ vs. $32.6 \pm 8.8$ years old; $p$ value $=0.01)$. No significant differences were found in BMI or in pre-supplementation levels of fasting plasma glucose, insulin, or plasma TG between high-risk and low-risk groups. There was a slight but significant difference in the plasma TG change between high-risk and low-risk groups $(-0.04 \pm$ 0.34 vs. $-0.27 \pm 0.47 \mathrm{mmol} / \mathrm{L}, p$ value $=0.01)$. This is in
Table 1. Characteristics of study participants classified as being at high risk and low risk of worsening insulin sensitivity following a 6-week n-3 PUFA supplementation

\begin{tabular}{|c|c|c|c|}
\hline Variable & High risk & Low risk & $p$ value \\
\hline Sex (men/women) & $15 / 17$ & $50 / 56$ & 0.98 \\
\hline TG response (yes/no) & $11 / 21$ & $38 / 68$ & 0.003 \\
\hline Age, years & $28.2 \pm 8.4$ & $32.6 \pm 8.8$ & 0.01 \\
\hline $\mathrm{BMI}, \mathrm{kg} / \mathrm{m}^{2}$ & $28.8 \pm 4.1$ & $28.2 \pm 3.5$ & 0.39 \\
\hline Glycemia, mmol/L & $4.88 \pm 0.53$ & $5.02 \pm 0.39$ & 0.11 \\
\hline Insulinemia, pmol/L & $91.0 \pm 55.0$ & $82.5 \pm 31.0$ & 0.27 \\
\hline Plasma TG, mmol/L & $1.41 \pm 0.73$ & $1.29 \pm 0.68$ & 0.39 \\
\hline$\Delta$ Plasma $\mathrm{TG}$ & $-0.04 \pm 0.34$ & $-0.27 \pm 0.47$ & 0.01 \\
\hline HOMA-IR (pre) & $2.83 \pm 2.05$ & $2.66 \pm 1.06$ & 0.49 \\
\hline HOMA-IR (post) & $4.20 \pm 1.76$ & $2.40 \pm 0.86$ & $3 \times 10^{-11}$ \\
\hline$\triangle$ HOMA-IR & $1.38 \pm 0.82$ & $-0.26 \pm 0.61$ & $<1 \times 10^{-15}$ \\
\hline
\end{tabular}

Data are means \pm standard deviations. Sex is presented as counts. $p$ values stand for two-sided Student's $t$ tests and $\chi^{2}$ tests (sex). BMI, body mass index; TG, triglycerides. TG response (yes/ no) stands for the number of TG responders and non-responders, as previously described [23]. Pre and post stand for presupplementation and postsupplementation.

line with the significantly higher proportion of high-risk subjects within the group of participants previously classified as TG nonresponders [16], as compared to low-risk subjects (65.6 vs. $34.4 \%$; $p$ value $=0.003$ ). Pre-supplementation HOMA-IR was similar in both high-risk and lowrisk groups, whereas post-supplementation HOMA-IR was significantly higher in high-risk participants (Table 1), concordant with the stratification applied. Therefore, a significantly higher HOMA-IR change was observed in the high-risk group, as compared to the low-risk group $\left(1.38 \pm 0.82\right.$ vs. $-0.26 \pm 0.61, p$ value $\left.<1 \times 10^{-15}\right)$ following the n-3 PUFA supplementation (Table 1).

\section{GWAS Revealed Novel SNPs Associated with Increased Insulin Resistance following n-3 PUFA Supplementation}

Results from the case/control association analysis between high-risk and low-risk groups showed that, after Bonferroni correction ( $p$ value $<1.87 \times 10^{-8}$ ), none of the SNPs analyzed was significantly associated with HOMAIR change in response to the n-3 PUFA supplementation. Yet, a total of 16 SNPs distributed across eight loci located on chromosomes $1,9,12,15,17$, and 22 were associated with HOMA-IR change at a suggestive genome-wide significance level ( $p$ value $<1 \times 10^{-5}$ ). After LD clumping, eight lead SNPs were identified and included in the construction of a GRS. Figure 2 highlights lead SNPs from 


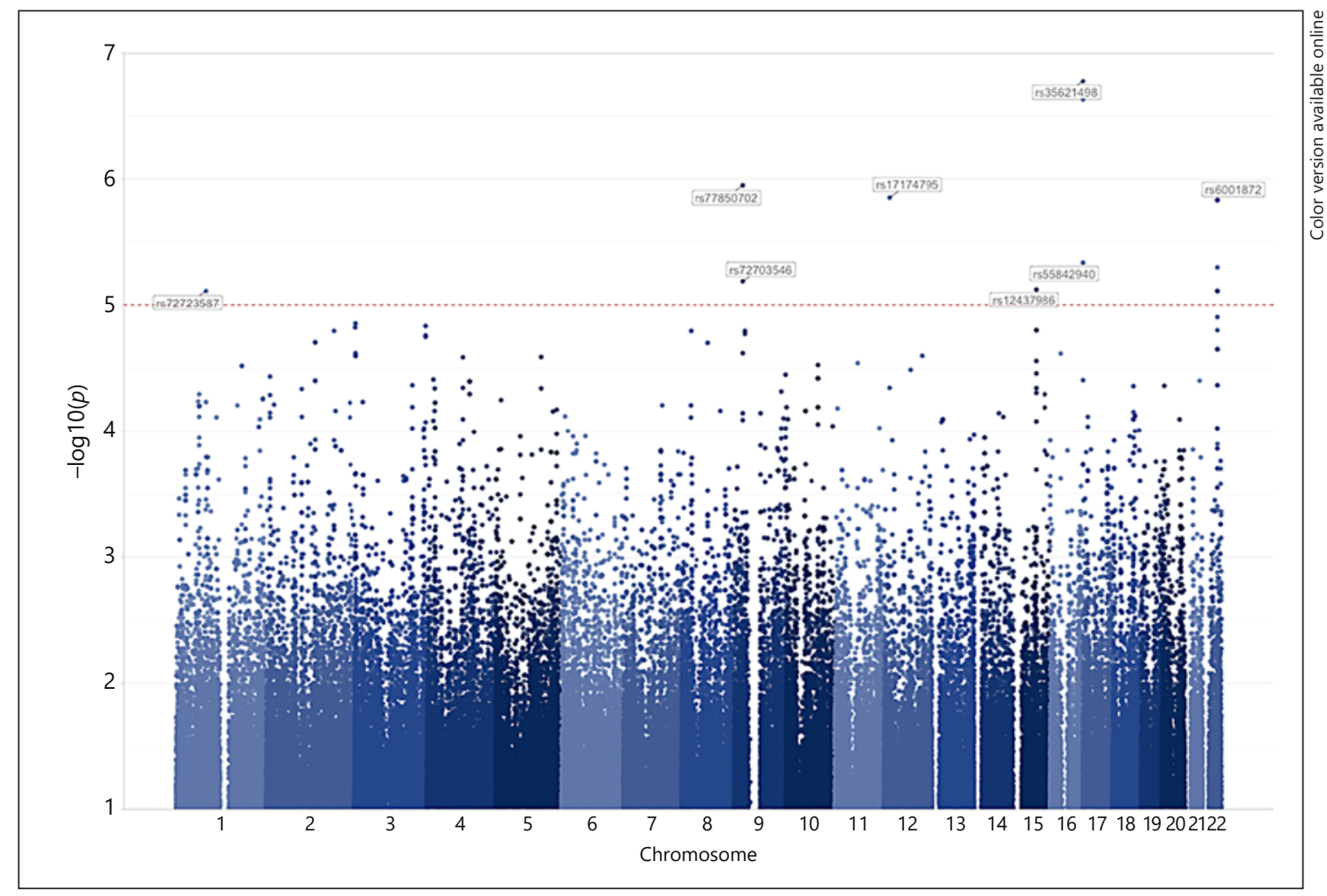

Fig. 2. GWAS results between high-risk and low-risk participants. Manhattan plot showing the negative logarithm of association $p$ values for each SNP with HOMA-IR risk status (HOMA-IR change following the n-3 PUFA supplementation) in participants from the FAS Study. A total of 8 loci at chromosomes 1, 9, 12, 15, 17, and 22 had significant frequency differences between high-risk and low-risk participants at a suggestive GWAS threshold ( $p$ value $<1 \times 10^{-5}$; red dashed line). Lead SNPs at each significant locus are shown in boxes.

Table 2. Description of lead SNPs showing suggestive significant association with HOMA-IR change following n-3 PUFA supplementation

\begin{tabular}{|c|c|c|c|c|c|c|c|c|c|}
\hline SNP & chr:bp & Mapping (nearest gene) & $\begin{array}{l}\text { Major } \\
\text { allele }\end{array}$ & $\begin{array}{l}\text { Minor } \\
\text { allele }\end{array}$ & \multicolumn{2}{|l|}{ MAF } & OR & $95 \%$ CI & $p$ value \\
\hline rs77850702 & $9: 23263040$ & Intergenic (LOC101929563) & $\mathrm{A}$ & G & 0.34 & 0.09 & 5.03 & $2.52-10.04$ & $1.13 \times 10^{-06}$ \\
\hline rs72703546 & $9: 23277588$ & Intergenic (LOC101929563) & $\mathrm{A}$ & $\mathrm{C}$ & 0.30 & 0.08 & 4.84 & $2.33-10.05$ & $6.45 \times 10^{-06}$ \\
\hline rs17174795 & $12: 15756489$ & Intergenic $(P T P R O)$ & A & $\mathrm{C}$ & 0.30 & 0.07 & 5.55 & $2.62-11.74$ & $1.41 \times 10^{-06}$ \\
\hline rs35621498 & $17: 158754$ & Intron $(R P H 3 A L)$ & $\mathrm{A}$ & G & 0.14 & 0.51 & 0.16 & $0.07-0.34$ & $1.67 \times 10^{-07}$ \\
\hline rs55842940 & $17: 160523$ & Intron $(R P H 3 A L)$ & G & $\mathrm{A}$ & 0.69 & 0.36 & 3.86 & $2.12-7.02$ & $4.60 \times 10^{-06}$ \\
\hline rs6001872 & $22: 40703245$ & Intron (TNRC6B) & $\mathrm{A}$ & G & 0.64 & 0.31 & 4.03 & $2.24-7.26$ & $1.47 \times 10^{-06}$ \\
\hline
\end{tabular}

SNP, single nucleotide polymorphism; chr:bp, chromosome and base-pair position of SNPs (genome build GRCh37/hg19); MAF, minor allele frequency in the high-risk and low-risk groups; OR, odds ratio; 95\% CI, 95\% confidence intervals; $p$ value, genome-wide association study $p$ value. 
loci having different frequencies between high-risk and low-risk participants at a suggestive genome-wide significance level ( $p$ value $<1 \times 10^{-5}$ ). Seven of these SNPs were associated with an increase in HOMA-IR following the n-3 PUFA supplementation, whereas a SNP located on chromosome 17 was associated with a decrease in HOMAIR. The complete list of lead SNPs of each loci associated with HOMA-IR is available in Table 2.

\section{The GRS Accurately Predicted Insulin Sensitivity}

Lowering following n-3 PUFA Supplementation

Finally, the eight lead SNPs associated with HOMA-IR change were included in the construction of the GRS. The GRS was computed by summing the number of alleles of these eight SNPs in each participant and ranged from -2 to 10 (Fig. 3a). Increasing GRS values indicate that a subject carries more at-risk allelic variants (with the minor at-risk allele being more represented in the high-risk group) from the SNPs identified in the GWAS (Fig. 3a). The predictive performance of the GRS was estimated after applying 10-fold cross-validation, resulting in an accuracy of 0.93 (95\% CI, 0.85-0.97) in the training dataset, and 0.85 (95\% CI, 0.73-0.93) in the testing dataset. The AUC-ROC also show a high predictive performance of the GRS in both the training (AUC-ROC $=0.97$ ) and testing dataset (AUC-ROC $=0.90$ ) (Fig. 3b). More precisely, the sensitivity and specificity, i.e. the proportion of highrisk and low-risk participants correctly identified as such with the GRS, respectively, were higher in the training dataset ( 0.80 and 0.97 , respectively), as compared to those found in the testing dataset ( 0.67 and 0.90 , respectively). The association between the GRS and HOMA-IR change was tested by binomial logistic regression and was found to be significant in both the training $(\mathrm{OR}=3.20[95 \% \mathrm{CI}$, 2.02-6.58]; Fig. 3c) and testing dataset ( $\mathrm{OR}=3.16[95 \%$ CI, 1.85-7.14]; Fig. 3d). The proportion of explained variation in HOMA-IR change was 0.69 in the training dataset (the subset of subjects used to fit the model), and 0.40 in the testing dataset (the subset of subjects used to evaluate the final model previously fit on the training dataset).

Fig. 3. Genetic risk score (GRS) was significantly associated with HOMA-IR change. a Density plots displaying the distribution of the GRS among low-risk participants (IS; grey) and high-risk participants (IR) in the training (orange) and testing (green) dataset. b The area under the receiver operating characteristic curve (AUCROC) of the GRS in the training and testing datasets. c, d Binomial logistic regression models with $95 \%$ confidence intervals (grey shade) for the likelihood of HOMA-IR increase following the

\section{Discussion/Conclusion}

The purpose of this study was to analyze the high interindividual variability observed in insulin sensitivity in response to an n-3 PUFA supplementation. The main finding is that participants being at high-risk of insulin sensitivity lowering following an n-3 PUFA supplementation may be identified using a genetic score approach.

First and foremost, HOMA-IR worsening was quite prevalent in both the FAS Study and the ComparED Study, emphasizing the deleterious effects of n-3 PUFA supplementation on insulin sensitivity in certain subjects, and the importance of identifying the origin of such metabolic deleterious effect. To this end, we compared wholegenome allele frequencies between subjects showing normal (low-risk) or impaired (high-risk) insulin sensitivity following an n-3 PUFA supplementation, and we identified eight suggestive genome-wide significant SNPs. This enabled us to construct a GRS in order to investigate whether its use could provide better information compared to individual SNPs. Evidence was provided that the GRS is predictive of increased HOMA-IR following the supplementation. The GRS had a significant association with the increased HOMA-IR even though only suggestive loci were identified by GWAS, pointing to a cumulative predictive power of the GRS over individual allelic variants. These results showed that genetic background may have a relevant role in the interindividual variability observed in the insulin sensitivity response following an n-3 PUFA supplementation.

As stated in the introduction, the cardioprotective effects of n-3 PUFA rely on pleiotropic pathways beyond a TG-lowering effect [18], among which only glycemic control was investigated in the present study. The effects of n-3 PUFA on glucose homeostasis and insulin sensitivity are controversial. Despite the beneficial effects observed in animal models [32-35], findings from human trials remain inconsistent. Indeed, a small number of studies reported either beneficial $[36,37]$ or deleterious effects $[38,39]$, while the majority yielded in-

n-3 PUFA supplementation in the training (orange) and testing (green) dataset, respectively. The $y$ axis represents the predicted probabilities for belonging to the IR (1) or to the IS group (0). Grey bars at the bottom of plots represent the distribution of the GRS across IS subjects. Orange and green bars at the top of plots represent the distribution of GRS across IR subjects in the training (c) and testing (d) dataset, respectively.

(For figure see next page.)

Insulin Sensitivity Lowering following an

n-3 PUFA Supplementation 

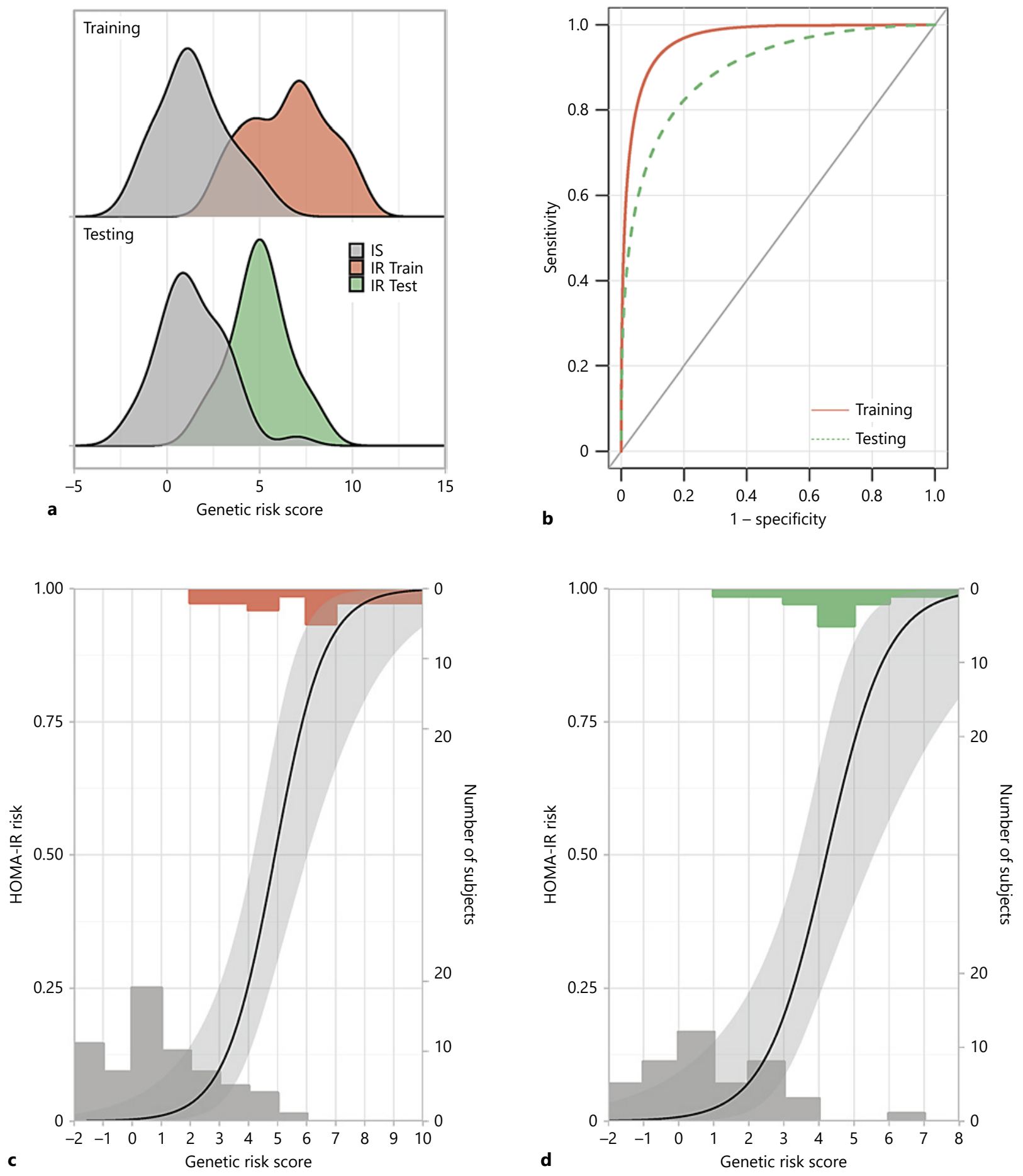
conclusive results [40-44]. This heterogeneity is supported by the interindividual variability observed in HOMA-IR response within the FAS Study, along with the heterogeneity demonstrated in the ComparED Study [22]. Consequently, given the inconsistency of previous studies, and in light of our findings, genetic background seems to be relevant in explaining the heterogeneity observed among studies and subjects. The discrepancies observed in the studies conducted in different ethnicities provide partial support for this assumption. Hence, in terms of insulin sensitivity, such studies pointed out that some populations seem to benefit more than others from a diet rich in n-3 PUFA [37, 45]. Furthermore, geographical differences in the frequency of SNPs related to key genes of n-3 PUFA metabolism have clearly been established, as demonstrated for the gene repertoire of FADS and ELOVL [4-7, 46], involved in n-3 PUFA desaturation and elongation pathways, respectively. Allelic variants of the FADS gene cluster have been associated with multiple risk factors; including inflammatory phenotypes, blood lipid levels, and notably insulin resistance $[25,47]$. As a matter of fact, genes of n-3 PUFA metabolism, such as FADS genes, are linked to metabolic and immune homeostasis, and had therefore been subjected and shaped by selective pressures conducted by the dietary environment. This has led to a genetic variability reflected by highly variable n-3 PUFA dietary needs, and so metabolic responses to n-3 PUFA $[2,48]$. Hence, this genetic heterogeneity among human beings seems to be evident when comparing populations which have been subjected to the founder effect, exposed to a homogeneous dietary environment and isolated. Though, it is present all over the world, but less palpable owing to migrations, interbreeding, and inconstant dietary exposure, forming this genetic interindividual variability which entails this heterogeneity in metabolic responses to n-3 PUFA. The present results show that the genetic background explains part of the heterogeneity observed in the insulin sensitivity response following an n-3 PUFA supplementation, as previously shown for the plasma TG response $[16,17]$. Thus, even though clinical studies have consistently shown a mean TG reduction following an n-3 PUFA supplementation, a substantial interindividual variability has also been noticed [22], which has been attributed, in part, to genetic background [17].

Conversely, although mean outcomes have been inconsistent regarding insulin sensitivity, the interindividual variability in the response to n-3 PUFA persists. Consequently, in the present study, while n-3 PUFA

Insulin Sensitivity Lowering following an n-3 PUFA Supplementation supplementation did not result in insulin sensitivity lowering for most of the participants, $23 \%$ of them exhibited a significant increase in HOMA-IR levels. Current lines of evidence are insufficient to promote $n-3$ PUFA supplementation for the purpose of improving and/or safeguarding insulin sensitivity $[12,13,49,50]$. In so doing, the results of the present study suggest that an n-3 PUFA supplementation may have, in some subjects, a deleterious impact on insulin sensitivity, and this is attributable, at least in part, to genetic background.

Previous GWAS have revealed a number of susceptibility variants for different traits and diseases, but of which over $90 \%$ are noncoding and can therefore not be readily assigned to an underlying biological function $[8$, 51, 52]. Moreover, mechanisms of blood glucose homeostasis are based on multiple genetic variants modulating various parameters, such as insulin secretion, clearance and sensitivity, as well as glucose conversion efficiency, with which n-3 PUFA appear to interact [21, $53,54]$.

Several SNPs located within the FADS gene cluster have been found to be associated with HOMA-IR, evidencing the link between n-3 PUFA intake, genes coding for key enzymes of n-3 PUFA metabolism and glucose homeostasis control [21, 47]. Taken together, and given that n-3 PUFA action is wide-ranging, dose-dependent, involving complex mechanisms, and interacting with dietary exposure, the metabolic pathways involved in glycemic control underlying the genetic variability herein observed remain elusive. Notwithstanding that GWAS findings reveal statistical associations but do not establish causation among the loci identified in the present study, polymorphisms within $R P H 3 A L$ may play a role in modulating the insulin sensitivity response to n-3 PUFA. Indeed, Noc2, which is encoded by the $R P H 3 A L$ gene, is essential to insulin exocytosis from pancreatic $\beta$-cells through its interaction with Rab-27A [55]. Moreover, the RAB27A gene, which codes for the Rab-27A protein, has been found to be upregulated following a 6-month n-3 PUFA supplementation (1.7 $\mathrm{g}$ of DHA and $0.6 \mathrm{~g}$ EPA) [56]. In any case, results of GWAS study do not constitute evidence of causality, but provide food for thought and guidance for further evaluation.

Risk SNPs involved in insulin sensitivity lowering generally derive from GWAS targeting allelic variants whose frequencies are statistically associated with diabetes [57-59]. With that said, the discovery of over 150 SNPs involved in the pathogenesis of type 2 diabetes [58- 
60] has led to the summation of numerous risk SNPs in GRS computation. In this way, several GRS have proved to be suitable tools in anticipating glucose homeostasis dysregulation and a decrease in insulin sensitivity, but irrespective of the plasticity of responses to environmental, and notably dietary exposure [61, 62]. Genes operate in networks rather than individually, and code for proteins involved in molecular and cellular mechanisms rather than for disease or dysfunction. This assumption provided direction to this project, employing GWAS methodology as a tool for the understanding of the adaptive glucose response to an environmental variation (n-3 PUFA supplementation), and building a GRS to increase the predictive power of individual allelic variants [14]. This is in line with the approach to personalize the implementation of nutritional interventions for complex traits and diseases, such as glucose homeostasis dysregulation and diabetes, according to genetic background [10]. Herein, the GRS of the present study was able to explain $40 \%$ of the variation in HOMA-IR change in response to an n-3 PUFA supplementation in the testing dataset, leaving a nonnegligible proportion of this variation unexplained, presumably due to multiple factors falling within the environment, genetic, epigenetic, or metagenomic signatures. By comparison, a previous GRS built in the FAS cohort by summing the number of risk alleles from GWAS hits was able to explain $21.5 \%$ of the variation in the TG response to an n-3 PUFA supplementation [16]. Thereafter, this GRS was refined by means of dense genotyping and genotype imputation, increasing the density of markers up to 31 SNPs, thus enhancing its predictive capacity and allowing to explain $49.7 \%$ of the variation in the TG response [19]. Interestingly, our findings also revealed a significantly higher proportion of high-risk participants within the group of TG nonresponders. In this respect, individuals combining both these deleterious features would appear to be likely to derive more harm than benefit from an n-3 PUFA supplementation, conversely to those TG responders showing low-risk features in terms of insulin sensitivity. A proper identification and stratification of such individuals thus represent a strategic approach towards improving personalized nutrition programs.

Some methodological considerations are relevant to mention in order to ensure a proper interpretation of the current findings. First, the use of TEM to classify participants into high-risk and low-risk groups constitutes a major strength of the present study, due to the increased reliability in the identification of actual atrisk participants [24]. The absence of an independent validation cohort has been addressed adequately by using cross-validation and by splitting the entire cohort into training and testing datasets, to appropriately fit and evaluate the model, respectively. However, it is worth highlighting that the difference observed in the explained variation in HOMA-IR change between the training and the testing datasets may be due to some degree of model overfitting, probably caused by the need to use the training dataset for SNP discovery purposes [63]. The sample size of the cohort on which the study relies, as well as the significance level of the loci brought to light, may also constitute limitations. Further validation of the GRS in larger and heterogeneous cohorts is clearly needed. At last, this GRS based on a GWAS of French Canadians could potentially misestimate the risk if applied to other populations, especially of non-European descent.

This study demonstrates that individuals with increased risk of insulin sensitivity lowering following an n-3 PUFA supplementation have a different genetic signature than those presenting no risk. Nonetheless, to what extent n-3 PUFA are linked to glucose homeostasis control, and from what allelic variants, still needs to be fully understood and assessed by the scientific community. In this respect, this study brings out to light the utility of a GRS as a tool to evaluate the effects of an n-3 PUFA supplementation in individuals carrying genetic variants that may influence insulin sensitivity.

\section{Acknowledgements}

The authors thank the study participants for their excellent collaboration and acknowledge the contribution of Catherine Raymond, Ann-Marie Paradis, Véronique Garneau and Élisabeth Thifault for laboratory work and technical assistance. The authors also thank the ComparED investigators (André Tchernof, Patrick Couture and Benoît Lamarche) for permission to use study data. J.T.M. received a postdoctoral fellowship from the Fonds de Recherche de Québec-Santé (FRQ-S). I.R. holds a Junior 2 Research Scholar from the FRQ-S. M.C.V. is Tier 1 Canada Research Chair in Genomics Applied to Nutrition and Metabolic Health.

\section{Disclosure Statement}

B.L. has received funding in the last 5 years from the Canadian Institutes of Health Research (CIHR) and Atrium Innovations, for which Douglas Laboratories manufacture and market omega-3 supplements, to study the effects of n-3 PUFA supplementation on cardiometabolic health. Douglas Laboratories provided the EPA, DHA, and control capsules used in the ComparED study.
40

Lifestyle Genomics 2020;13:32-42 DOI: $10.1159 / 000504022$
Franck et al. 


\section{Statement of Ethics}

The study has been approved by the Ethics Committees of Laval University Hospital Research Center and Laval University, and has been performed in accordance with the principles of the Declaration of Helsinki. All participants signed a written informed consent before initiation of the study.

\section{Funding Sources}

This work was partly supported by an operating grant from the CIHR (MOP-110975). The financial support for the ComparED Study was provided via a grant from the CIHR (MOP-123494).

\section{Author Contributions}

J.T.M. and M.F. interpreted data; M.F. drafted the manuscript; J.T.M. performed statistical analysis and major revision of the manuscript; F.G. was in charge of data management. S.L. and M.C.V. designed the research; P.C. was responsible for the medical follow-up. F.G., I.R., S.L., B.L., P.C., and M.C.V. critically revised the manuscript. All authors read and approved the final version of the manuscript. M.F., J.T.M., and M.C.V. have primary responsibility for final content.

\section{References}

1 Uribe-Salazar JM, Palmer JR, Haddad SA, Rosenberg L, Ruiz-Narváez EA. Admixture mapping and fine-mapping of type 2 diabetes susceptibility loci in African American women. J Hum Genet. 2018 Nov;63(11):1109-17.

2 Chilton FH, Dutta R, Reynolds LM, Sergeant S, Mathias RA, Seeds MC. Precision Nutrition and Omega-3 Polyunsaturated Fatty Acids: A Case for Personalized Supplementation Approaches for the Prevention and Management of Human Diseases. Nutrients. 2017 Oct; 9(11):E1165.

3 Vallée Marcotte B, Guénard F, Marquis J, Charpagne A, Vadillo-Ortega F, Tejero ME, et al. Genetic Risk Score Predictive of the Plasma Triglyceride Response to an Omega-3 Fatty Acid Supplementation in a Mexican Population. Nutrients. 2019 Mar;11(4):E737.

4 Mathias RA, Sergeant S, Ruczinski I, Torgerson DG, Hugenschmidt CE, Kubala M, et al. The impact of FADS genetic variants on $\omega 6$ polyunsaturated fatty acid metabolism in African Americans. BMC Genet. 2011 May; 12(1):50.

5 Ameur A, Enroth S, Johansson A, Zaboli G, Igl W, Johansson AC, et al. Genetic adaptation of fatty-acid metabolism: a human-specific haplotype increasing the biosynthesis of long-chain omega- 3 and omega- 6 fatty acids. Am J Hum Genet. 2012 May;90(5):809-20.

6 Fumagalli M, Moltke I, Grarup N, Racimo F, Bjerregaard P, Jørgensen ME, et al. Greenlandic Inuit show genetic signatures of diet and climate adaptation. Science. 2015 Sep; 349(6254):1343-7.

7 Lemaitre RN, Tanaka T, Tang W, Manichaikul A, Foy M, Kabagambe EK, et al. Genetic loci associated with plasma phospholipid n-3 fatty acids: a meta-analysis of genome-wide association studies from the CHARGE Consortium. PLoS Genet. 2011 Jul;7(7):e1002193.

8 Barroso I, McCarthy MI. The Genetic Basis of Metabolic Disease. Cell. 2019 Mar;177(1): 146-61.

9 Kotas ME, Medzhitov R. Homeostasis, inflammation, and disease susceptibility. Cell. 2015 Feb;160(5):816-27.
10 de Toro-Martín J, Arsenault BJ, Després JP, Vohl MC. Precision Nutrition: A Review of Personalized Nutritional Approaches for the Prevention and Management of Metabolic Syndrome. Nutrients. 2017 Aug;9(8):E913.

11 Molfino A, Amabile MI, Monti M, Muscaritoli M. Omega-3 Polyunsaturated Fatty Acids in Critical Illness: Anti-Inflammatory, Proresolving, or Both? Oxid Med Cell Longev. 2017;2017:5987082.

12 Albracht-Schulte K, Kalupahana NS, Ramalingam L, Wang S, Rahman SM, Robert-McComb J, et al. Omega-3 fatty acids in obesity and metabolic syndrome: a mechanistic update. J Nutr Biochem. 2018 Aug;58:1-16.

13 Li D. Omega-3 polyunsaturated fatty acids and non-communicable diseases: meta-analysis based systematic review. Asia Pac J Clin Nutr. 2015;24(1):10-5.

14 Galmés S, Cifre M, Palou A, Oliver P, Serra F. A Genetic Score of Predisposition to LowGrade Inflammation Associated with Obesity May Contribute to Discern Population at Risk for Metabolic Syndrome. Nutrients. 2019 Jan; 11(2):E298.

15 Caslake MJ, Miles EA, Kofler BM, Lietz G, Curtis P, Armah CK, et al. Effect of sex and genotype on cardiovascular biomarker response to fish oils: the FINGEN Study. Am J Clin Nutr. 2008 Sep;88(3):618-29.

16 Rudkowska I, Guénard F, Julien P, Couture P, Lemieux S, Barbier O, et al. Genome-wide association study of the plasma triglyceride response to an n-3 polyunsaturated fatty acid supplementation. J Lipid Res. 2014 Jul;55(7): 1245-53.

17 Vallée Marcotte B, Cormier H, Guénard F, Rudkowska I, Lemieux S, Couture P, et al. Novel Genetic Loci Associated with the Plasma Triglyceride Response to an Omega-3 Fatty Acid Supplementation. J Nutrigenet Nutrigenomics. 2016;9(1):1-11.

18 Bhatt DL, Steg PG, Brinton EA, Jacobson TA, Miller M, Tardif JC, et al.; REDUCE-IT Investigators. Rationale and design of REDUCEIT: Reduction of Cardiovascular Events with
Icosapent Ethyl-Intervention Trial. Clin Cardiol. 2017 Mar;40(3):138-48.

19 Vallée Marcotte B, Guénard F, Lemieux S, Couture P, Rudkowska I, Calder PC, et al. Fine mapping of genome-wide association study signals to identify genetic markers of the plasma triglyceride response to an omega-3 fatty acid supplementation. Am J Clin Nutr. 2019 Jan;109(1):176-85.

20 Telle-Hansen VH, Gaundal L, Myhrstad MC. Polyunsaturated Fatty Acids and Glycemic Control in Type 2 Diabetes. Nutrients. 2019 May;11(5):E1067.

21 Cormier H, Rudkowska I, Thifault E, Lemieux S, Couture P, Vohl MC. Polymorphisms in Fatty Acid Desaturase (FADS) Gene Cluster: Effects on Glycemic Controls Following an Omega-3 Polyunsaturated Fatty Acids (PUFA) Supplementation. Genes (Basel). 2013 Sep;4(3):485-98.

22 Allaire J, Couture P, Leclerc M, Charest A, Marin J, Lépine MC, et al. A randomized, crossover, head-to-head comparison of eicosapentaenoic acid and docosahexaenoic acid supplementation to reduce inflammation markers in men and women: the Comparing EPA to DHA (ComparED) Study. Am J Clin Nutr. 2016 Aug;104(2):280-7.

23 Rudkowska I, Paradis AM, Thifault E, Julien $\mathrm{P}$, Tchernof A, Couture P, et al. Transcriptomic and metabolomic signatures of an n-3 polyunsaturated fatty acids supplementation in a normolipidemic/normocholesterolemic Caucasian population. J Nutr Biochem. 2013 Jan;24(1):54-61.

24 de Lannoy L, Clarke J, Stotz PJ, Ross R. Effects of intensity and amount of exercise on measures of insulin and glucose: analysis of interindividual variability. PLoS One. 2017 May; 12(5):e0177095.

25 de Toro-Martín J, Guenard F, Rudkowska I, Lemieux S, Couture P, Vohl MC. A common variant in ARHGEF10 alters delta-6 desaturase activity and influence susceptibility to hypertriglyceridemia. J Clin Lipidol. 2018; 12(2):311-20 e3. 
26 Chang CC, Chow CC, Tellier LC, Vattikuti S, Purcell SM, Lee JJ. Second-generation PLINK: rising to the challenge of larger and richer datasets. Gigascience. 2015 Feb;4(1):7.

27 Lander E, Kruglyak L. Genetic dissection of complex traits: guidelines for interpreting and reporting linkage results. Nat Genet. 1995 Nov;11(3):241-7.

28 Kuhn M. Building Predictive Models in R Using the caret Package. J Stat Softw. 2008;28(5).

29 Kuhn M. caret: Classification and Regression Training. R package version 6.0-84. 2019.

$30 \mathrm{McF}$ adden D. Conditional logit analysis of qualitative choice behavior. In: Zarembka $\mathrm{P}$, editor. Frontiers in Econometrics. New York: Academic Press; 1974.

31 R Core Team. R: A language and environment for statistical computing. Vienna, Austria: R Foundation for Statistical Computing; 2018.

32 Bremer AA, Stanhope KL, Graham JL, Cummings BP, Ampah SB, Saville BR, et al. Fish oil supplementation ameliorates fructose-induced hypertriglyceridemia and insulin resistance in adult male rhesus macaques. J Nutr. 2014 Jan;144(1):5-11.

33 Pérez-Matute P, Pérez-Echarri N, Martínez JA, Marti A, Moreno-Aliaga MJ. Eicosapentaenoic acid actions on adiposity and insulin resistance in control and high-fat-fed rats: role of apoptosis, adiponectin and tumour necrosis factor-alpha. Br J Nutr. 2007 Feb;97(2): 389-98.

34 Mayer C, Côme M, Ulmann L, Chini Zittelli G, Faraloni C, Nazih H, et al. Preventive Effects of the Marine Microalga Phaeodactylum tricornutum, Used as a Food Supplement, on Risk Factors Associated with Metabolic Syndrome in Wistar Rats. Nutrients. 2019 May; 11(5):E1069.

35 Storlien LH, Kraegen EW, Chisholm DJ, Ford GL, Bruce DG, Pascoe WS. Fish oil prevents insulin resistance induced by high-fat feeding in rats. Science. 1987 Aug;237(4817):885-8.

36 Popp-Snijders C, Schouten JA, Heine RJ, van der Meer J, van der Veen EA. Dietary supplementation of omega-3 polyunsaturated fatty acids improves insulin sensitivity in non-insulin-dependent diabetes. Diabetes Res. 1987 Mar;4(3):141-7.

37 Paquet C, Propsting SL, Daniel M. Total n-3 fatty acid and SFA intakes in relation to insulin resistance in a Canadian First Nation at risk for the development of type 2 diabetes. Public Health Nutr. 2014 Jun;17(6):1337-41.

38 Vessby B, Boberg M. Dietary supplementation with n-3 fatty acids may impair glucose homeostasis in patients with non-insulin-dependent diabetes mellitus. J Intern Med. 1990 Aug;228(2):165-71.

39 Glauber H, Wallace P, Griver K, Brechtel G. Adverse metabolic effect of omega-3 fatty acids in non-insulin-dependent diabetes mellitus. Ann Intern Med. 1988 May;108(5):6638.

40 Giacco R, Cuomo V, Vessby B, Uusitupa M, Hermansen K, Meyer BJ, et al.; KANWU Study Group. Fish oil, insulin sensitivity, in- sulin secretion and glucose tolerance in healthy people: is there any effect of fish oil supplementation in relation to the type of background diet and habitual dietary intake of n-6 and n-3 fatty acids? Nutr Metab Cardiovasc Dis. 2007 Oct;17(8):572-80.

41 Gulseth HL, Gjelstad IM, Tiereny AC, McCarthy D, Lovegrove JA, Defoort C, et al. Effects of dietary fat on insulin secretion in subjects with the metabolic syndrome. Eur J Endocrinol. 2019 May;180(5):321-8.

42 Browning LM, Krebs JD, Moore CS, Mishra GD, O'Connell MA, Jebb SA. The impact of long chain $n-3$ polyunsaturated fatty acid supplementation on inflammation, insulin sensitivity and CVD risk in a group of overweight women with an inflammatory phenotype. Diabetes Obes Metab. 2007 Jan;9(1):70-80.

43 Clark LF, Thivierge MC, Kidd CA, McGeoch SC, Abraham P, Pearson DW, et al. Fish oil supplemented for 9 months does not improve glycaemic control or insulin sensitivity in subjects with impaired glucose regulation: a parallel randomised controlled trial. $\mathrm{Br} \mathrm{J}$ Nutr. 2016 Jan;115(1):75-86.

44 O’Mahoney LL, Matu J, Price OJ, Birch KM, Ajjan RA, Farrar D, et al. Omega-3 polyunsaturated fatty acids favourably modulate cardiometabolic biomarkers in type 2 diabetes: a meta-analysis and meta-regression of randomized controlled trials. Cardiovasc Diabetol. 2018 Jul;17(1):98.

45 Thorseng T, Witte DR, Vistisen D, BorchJohnsen K, Bjerregaard P, Jørgensen ME. The association between $\mathrm{n}-3$ fatty acids in erythrocyte membranes and insulin resistance: the Inuit Health in Transition Study. Int J Circumpolar Health. 2009 Sep;68(4):327-36.

46 Harris DN, Ruczinski I, Yanek LR, Becker LC, Becker DM, Guio H, et al. Evolution of Hominin Polyunsaturated Fatty Acid Metabolism: From Africa to the New World. Genome Biol Evol. 2019 May;11(5):1417-30.

47 Brayner B, Kaur G, Keske MA, Livingstone KM. FADS Polymorphism, Omega-3 Fatty Acids and Diabetes Risk: A Systematic Review. Nutrients. 2018 Jun;10(6):E758.

48 Chen Y, Estampador AC, Keller M, Poveda A, Dalla-Riva J, Johansson I, et al. The combined effects of FADS gene variation and dietary fats in obesity-related traits in a population from the far north of Sweden: the GLACIER Study. Int J Obes. 2019 Apr;43(4):808-20.

49 Lalia AZ, Lanza IR. Insulin-Sensitizing Effects of Omega-3 Fatty Acids: lost in Translation? Nutrients. 2016 Jun;8(6):E329.

50 Chen C, Yu X, Shao S. Effects of Omega-3 Fatty Acid Supplementation on Glucose Control and Lipid Levels in Type 2 Diabetes: A MetaAnalysis. PLoS One. 2015 Oct; 10(10): e0139565.

51 Edwards SL, Beesley J, French JD, Dunning AM. Beyond GWASs: illuminating the dark road from association to function. Am J Hum Genet. 2013 Nov;93(5):779-97.

52 Visscher PM, Wray NR, Zhang Q, Sklar P, McCarthy MI, Brown MA, et al. 10 Years of
GWAS Discovery: Biology, Function, and Translation. Am J Hum Genet. 2017 Jul; 101(1):5-22.

53 Vallée Marcotte B, Cormier H, Rudkowska I, Lemieux S, Couture P, Vohl MC. Polymorphisms in FFAR4 (GPR120) Gene Modulate Insulin Levels and Sensitivity after Fish Oil Supplementation. J Pers Med. 2017 Nov; 7(4):E15.

54 Röder PV, Wu B, Liu Y, Han W. Pancreatic regulation of glucose homeostasis. Exp Mol Med. 2016 Mar;48(3):e219.

55 Matsunaga K, Taoka M, Isobe T, Izumi T. Rab2a and Rab27a cooperatively regulate the transition from granule maturation to exocytosis through the dual effector Noc2. J Cell Sci. 2017 Feb;130(3):541-50.

56 Vedin I, Cederholm T, Freund-Levi Y, Basun $\mathrm{H}$, Garlind A, Irving GF, et al. Effects of DHArich n-3 fatty acid supplementation on gene expression in blood mononuclear leukocytes: the OmegAD study. PLoS One. 2012;7(4): e35425.

57 Suzuki K, Akiyama M, Ishigaki K, Kanai M, Hosoe J, Shojima N, et al. Identification of 28 new susceptibility loci for type 2 diabetes in the Japanese population. Nat Genet. 2019 Mar;51(3):379-86.

58 Dupuis J, Langenberg C, Prokopenko I, Saxena R, Soranzo N, Jackson AU, et al.; DIAGRAM Consortium; GIANT Consortium; Global BPgen Consortium; Anders Hamsten on behalf of Procardis Consortium; MAGIC investigators. New genetic loci implicated in fasting glucose homeostasis and their impact on type 2 diabetes risk. Nat Genet. 2010 Feb; 42(2):105-16.

59 Scott RA, Scott LJ, Mägi R, Marullo L, Gaulton KJ, Kaakinen M, et al.; DIAbetes Genetics Replication And Meta-analysis (DIAGRAM) Consortium. An Expanded Genome-Wide Association Study of Type 2 Diabetes in Europeans. Diabetes. 2017 Nov;66(11):2888902.

60 Palmer ND, Goodarzi MO, Langefeld CD Wang N, Guo X, Taylor KD, et al. Genetic Variants Associated With Quantitative Glucose Homeostasis Traits Translate to Type 2 Diabetes in Mexican Americans: The GUARDIAN (Genetics Underlying Diabetes in Hispanics) Consortium. Diabetes. 2015 May;64(5):1853-66.

61 Stančáková A, Kuulasmaa T, Kuusisto J, Mohlke KL, Collins FS, Boehnke M, et al. Genetic risk scores in the prediction of plasma glucose, impaired insulin secretion, insulin resistance and incident type 2 diabetes in the METSIM study. Diabetologia. 2017 Sep; 60(9):1722-30.

62 Ericson U, Hindy G, Drake I, Schulz CA, Brunkwall L, Hellstrand S, et al. Dietary and genetic risk scores and incidence of type $2 \mathrm{di}$ abetes. Genes Nutr. 2018 May;13(1):13.

63 Cawley GC, Talbot NL. On Over-fitting in Model Selection and Subsequent Selection Bias in Performance Evaluation. J Mach Learn Res. 2010;11:2079-107. 